\title{
Bio-Click Chemistry: Enzymatic Functionalization of PEGylated Capsules for Targeting Applications
}

\author{
Melissa K. M. Leung, Christoph E. Hagemeyer, Angus P. R. Johnston, Catalina Gonzales, Marloes M. \\ J. Kamphuis, Katie Ardipradja, Georgina K. Such, Karlheinz Peter, and Frank Caruso*
}

Targeted delivery with nanoengineered biofunctionalized pharmaceutical carriers has the potential to maximize the uptake of drugs at a diseased site while minimizing therapeutic side effects. ${ }^{[1]}$ Effective drug delivery can be significantly enhanced through covalent and site-specific protein immobilization on the carrier vehicles, as it avoids random orientation and inactivation of celltargeting ligands/antibodies. Although covalent biofunctionalization can be readily performed on protein functional groups using carbodiimide and maleimide chemistries, these methods do not provide site-directed attachment due to the abundance of protein functional groups. Advanced techniques such as click chemistry have been commonly used for site-specific immobilization; ${ }^{[2]}$ however, it requires the incorporation of synthetic functional groups into biomolecules. More recently it has been demonstrated that antibodies expressed as fusion proteins with a SNAP-tag $\left(\mathrm{O}_{6^{-}}\right.$ alkylguanine-DNA alkyltransferase $)^{[3 a]}$ can be coupled to $\mathrm{O}_{6^{-}}$

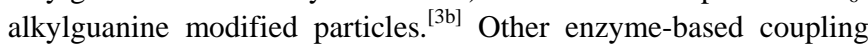
approaches have also been reported for site-selective conjugation but are limited by large-sized protein fusions and multiple preparation steps. ${ }^{[4]}$ Hence, there remains a need for an efficient method for the covalent and site-specific functionalization of drug carriers.

A relatively new tool for bioconjugation is based on the Staphylococcus aureus enzyme Sortase A (Srt A), which covalently

[*] M. K. M. Leung, Dr. A. P. R. Johnston, C. Gonzales, M. M. J. Kamphuis, Dr. G. K. Such, Prof. F. Caruso Department of Chemical and Biomolecular Engineering The University of Melbourne, Victoria 3010 (Australia) Fax: (+61) 3-8344-4153

E-mail: fcaruso@unimelb.edu.au

Dr. C. E. Hagemeyer, K. Ardipradja, Prof. K. Peter Atherothrombosis and Vascular Biology Baker IDI Heart and Diabetes Institute Melbourne, Victoria 3004 (Australia)

Dr. C. E. Hagemeyer, Prof. K. Peter

Departments of Medicine (K.P.), Clinical Haematology (C.E.H.) and Immunology (K.P., C.E.H.) Monash University, Victoria 3800 (Australia)

[**] The authors are supported by the Australian Research Council via an Australian Postgraduate Award (M.K.M.L.), Discovery Projects (A.P.R.J., G.K.S., F.C.), a Future Fellowship (A.P.R.J., K.P.) and a Federation Fellowship (F.C.). Financial support was also provided by the National Health and Medical Research Council under the Program Grants 472665 (K.P.), 487922 (F.C.) and 1017670 (K.P.) and Career Development Award (C.E.H.), and in part by the Victorian Government's Operational Infrastructure Support Program.

Supporting information for this article is available on the WWW under http://www.angewandte.org or from the author. attaches proteins to the bacterial cell wall. ${ }^{[5]}$ Srt A recognizes protein substrates that contain an LPETG peptide motif and cleaves between the threonine and glycine residues, where the carboxyl group of threonine subsequently undergoes a nucleophilic attack by the N-terminal amino group of a polyglycine, forming an amide bond. ${ }^{[6]}$ Since its discovery, Srt A has been used to synthesize peptide conjugates with sugars, ${ }^{[7]}$ glycolipids ${ }^{[8]}$ and peptide nucleic acids, ${ }^{[9]}$ which are typically difficult to obtain by chemical reactions. The application of Srt A has also been extended to in vitro and in vivo studies for labeling proteins on living cell surfaces and molecular imaging. ${ }^{[10]}$ Recently, the enzyme has been employed to immobilize proteins on solid supports; ${ }^{[11]}$ however, these studies have not been aimed at the development of therapeutic delivery systems for targeting applications. Herein, we report the use of Srt A as a "bio-click" approach for the functionalization of polymer drug vehicles. This enzymatic method possesses many of the useful properties of click chemistry; namely, it is highly specific, efficient, proceeds under mild conditions and undergoes no unwanted side reactions.

A phage-display derived single single-chain variable fragment (scFv) was used as a model antibody to demonstrate the Srt Acatalyzed functionalization of polymer capsules and subsequent targeting. This class of antibody fragment consists only of the variable regions of immunoglobulins and has the same targeting specificity of the parental form. Nonetheless, due to their small size, scFvs have low immunogenicity with more efficient tissue penetration, and thus have advantages over whole antibodies for targeting applications. ${ }^{[12]}$ Moreover, genetic engineering allows easy introduction of functional residues into scFvs, making them suitable for site-specific coupling. The $\mathrm{scFv}$ used in this work has been shown to bind selectively to the activated glycoprotein (GP) IIb/IIIa receptor on platelets, ${ }^{[13]}$ which in turn aggregate via GPIIb/IIIa to form thrombi. Since platelet aggregation plays a critical role in thrombosis, GPIIb/IIIa presents a therapeutic target for the disease. ${ }^{[13]}$ To make this scFv a suitable substrate for Srt A, it was encoded with an LPETG sequence (scFv-LPETG) at the C-terminus, which is not within the targeting regions of the $\mathrm{scFv}$, thereby preserving its bioactivity. In addition, this ScFv was also cloned with an $\mathrm{H}_{6}$ tag after the LPETG motif for purification, which can be later cleaved off during the enzymatic ligation. Hence, Srt A mediates both the coupling and removal of the purification tag in one single step.

To develop scFv-functionalized delivery vehicles, low-fouling capsules assembled by the layer-by-layer (LbL) technique were used as the carrier system. Low-fouling materials such as poly(ethylene glycol) (PEG) and poly( $N$-vinyl pyrrolidone) (PVPON) are of interest for drug delivery as they exhibit low nonspecific binding to cells. The capsules were assembled through hydrogen bonding by alternately layering alkyne-modified (1\%) PVPON (PVPON $\left.{ }_{\mathrm{Alk}}\right)^{[14]}$ and poly(methacrylic acid) PMA on silica particles $(\sim 3 \mu \mathrm{m}$ diameter), followed by deposition of alkyne-modified (5\%) PEG $\left(\mathrm{PEG}_{\mathrm{Alk}}\right)^{[15]}$ for PEGylation (Figure 1). The alkyne groups of the 
multilayer were then cross-linked with a bisazide linker ${ }^{[14]}$ via alkyne-azide click chemistry. To immobilize scFv-LPETG on the PEG $_{\text {Alk }}$ surface, using click chemistry, the particles were functionalized with an azide-modified triglycine. This peptide is a bifunctional PEG linker (4 PEG units) terminated with a GGGWWSSK sequence and an azide (GGG-PEG-Az), where azidemodified PEG is coupled to the lysine residue at the C-terminus. Subsequently, the GGG-PEG-Az-modified particles were capped with sacrificial hydrogen bonded PVPON/PMA films to impart colloidal stability to the particles upon core dissolution in hydrofluoric acid (HF). It was found that without the capping layers, the peptide-coated capsules aggregated in the presence of HF (data not shown). After core removal, the capsules were exposed to $\mathrm{pH} 7$ to remove PVPON and PMA by disrupting the hydrogen bonds, forming GGG-PEG-Az-modified PVPON $_{\mathrm{Alk}} / \mathrm{PEG}_{\mathrm{Alk}}$ capsules. Finally, scFv-LPETG was conjugated to the capsules in the presence of Srt A to yield scFv-functionalized PEGylated capsules.

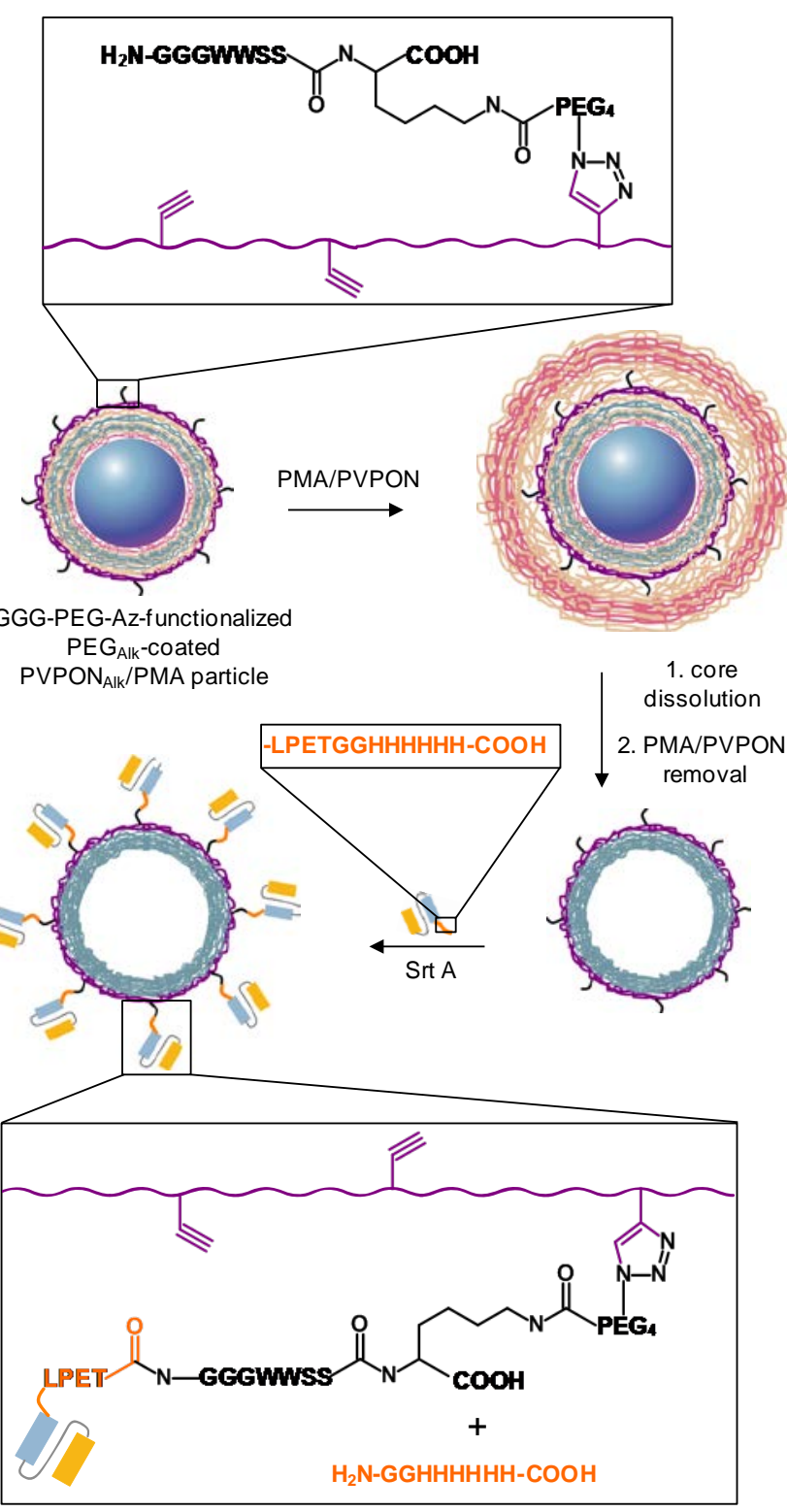

Figure 1. Preparation of ScFv-functionalized PEGylated $\mathrm{PVPON}_{\mathrm{Alk}}$

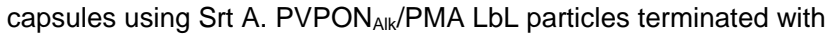
$\mathrm{PEG}_{\mathrm{Alk}}$ were functionalized with GGG-PEG-Az, followed by adsorption of protective capping layers of PMA/PVPON. The silica templates were then dissolved in HF and the resulting capsules were exposed to $\mathrm{pH} 7$ to release PMA and PVPON. The GGG-PEG-Az-coated $P V P O N_{A \mid k} / P E G_{A l k}$ capsules were finally incubated with scFv-LPETG in the presence of Srt A, yielding scFv-functionalized capsules (where the scFv purification tag was removed).

The immobilization of scFv-LPETG on multilayer films was first followed on planar supports using quartz crystal microgravimetry (QCM) (Figure 2). Preliminary studies were conducted on a $\mathrm{PEG}_{\text {Alk }}$-terminating film as a model system, where PEG $_{\text {Alk }}$ was hydrogen bonded to a poly(ethylenimine)

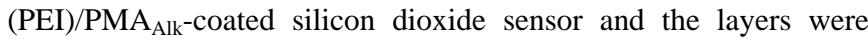
cross-linked with the bisazide linker. GGG-PEG-Az was then covalently coupled to the $\mathrm{PEG}_{\text {Alk }}$ surface by the $\mathrm{Cu}(\mathrm{I})$-catalyzed cycloaddition. In these studies, the ratio of dissipation to frequency was high $\left(>1 \times 10^{6}\right.$ per $\left.10 \mathrm{~Hz}\right)$; thus the Sauerbrey equation is not valid. ${ }^{[16]}$ However, the dissipation/frequency ratio remained similar throughout the experiments, hence a change in frequency is indicative of a change in mass. In the presence of $\mathrm{Cu}(\mathrm{I})$, a frequency change of $-17.7 \mathrm{~Hz}$ was obtained for the attachment of GGG-PEG$\mathrm{Az}$ while there was no significant frequency change in the absence of the catalyst (Figure 2a). This indicates the critical role of $\mathrm{Cu}(\mathrm{I})$ as the catalyst to promote the covalent linkages of GGG-PEG-Az to the PEG $_{\text {Alk }}$ surface, making the films a suitable substrate for the subsequent enzymatic coupling.
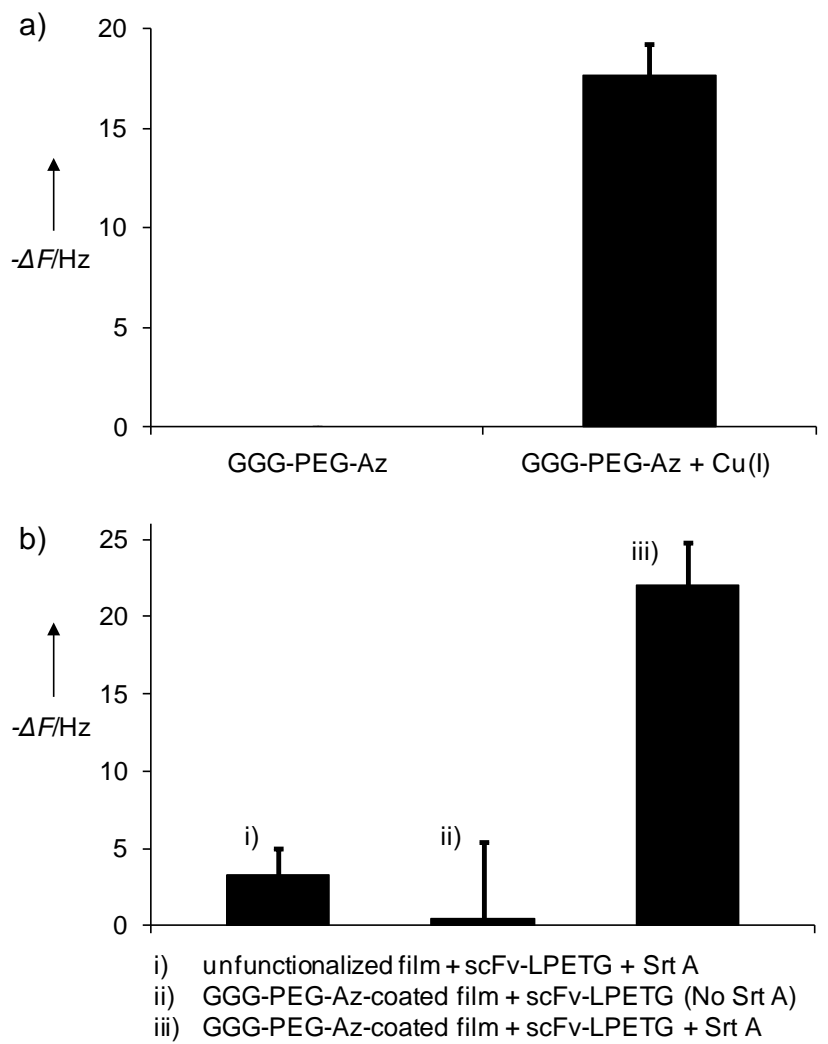

Figure 2. Frequency change $(\Delta F)$ for the functionalization of $P E G_{A l k}-$ terminating films with GGG-PEG-Az and scFv-LPETG, monitored by QCM. a) $P E G_{A l k}$-terminating films were modified with GGG-PEG-Az in the absence and presence of $\mathrm{Cu}(\mathrm{I})$ by click chemistry for $30 \mathrm{~min}$ in $\mathrm{pH}$ 5 sodium acetate $(150 \mathrm{mM})$. b) ScFv-LPETG was immobilized on i) the unfunctionalized films in the presence of Srt A, and on the GGGPEG-Az-coated films in the ii) absence and iii) presence of Srt $A$. The films were incubated at $0.1 \mathrm{~g} \mathrm{~L}^{-1}$ of Srt A and scFv-LPETG at $37^{\circ} \mathrm{C}$ for $1 \mathrm{~h}$ at $\mathrm{pH} 8$ (50 mM tris(hydroxymethyl)aminomethane (Tris), 150 $\mathrm{mM}$ sodium chloride $(\mathrm{NaCl})$ ). 
Next, the GGG-PEG-Az-coated and uncoated films were functionalized with scFv-LPETG in a pH 8 buffer solution at $37^{\circ} \mathrm{C}$. After $1 \mathrm{~h}$ incubation, there was no further change in frequency and the films were washed with the buffer to remove any unbound scFv and the frequency change was measured. The Srt A-catalyzed coupling of scFv-LPETG resulted in a frequency change of -22.0 $\mathrm{Hz}$ on the GGG-PEG-Az-modified film (Figure 2b, iii). In the absence of Srt A, no significant frequency change was seen $(-0.5$ $\mathrm{Hz}$ ) on the triglycine-coated surface (Figure 2b, ii). When the scFv was deposited on the unfunctionalized film, the adsorption was low $(-3.3 \mathrm{~Hz})$ even in the presence of the enzyme (Figure 2b, i). These studies confirm that the conjugation of scFv-LPETG to the GGGPEG-Az-modified films was specifically catalyzed by Srt A. Furthermore, the low nonspecific binding of the scFv to the functionalized/unfunctionalized surfaces via physical adsorption revealed the low-fouling properties of the PEGylated films. This Srt A-mediated immobilization technique provides a platform for fast $(<1 \mathrm{~h})$, covalent and site-specific functionalization of polymer films with biomolecules in mild conditions ( $\mathrm{pH} 8$ ).

The immobilization of scFv-LPETG was also performed on capsules under the conditions investigated above. Functionalization of $\mathrm{PVPON}_{\mathrm{Alk}} / \mathrm{PEG}_{\mathrm{Alk}}$ capsules with the $\mathrm{scFv}$ was verified using fluorescently labeled scFv (Figure 3) and the amount of conjugated $\mathrm{scFv}$ was quantified using fluorescence spectroscopy. It was determined that approximately $0.36 \mu \mathrm{g}$ of protein was coupled to $2.6 \times 10^{6}$ capsules, corresponding to $3 \times 10^{6} \mathrm{scFv}$ per capsule. This suggests that the $\mathrm{scFv}$ is densely packed on the surface of the capsules, which is in agreement with our previous study on the antibody functionalization of capsules using click chemistry. ${ }^{\text {[2a] }}$ While copper-catalyzed azide-alkyne cycloaddition (CuAAC) and sortase-mediated functionalization both afford similar coupling densities, each method offers its own advantages. CuAAC coupling is a convenient method for attaching whole antibodies to surfaces, whereas sortase-mediated functionalization is suited for coupling engineered antibody fragments, such as scFv, to surfaces with a controlled and defined orientation.

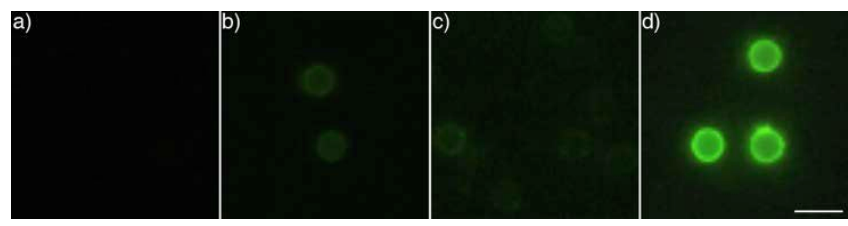

Figure 3. Fluorescence microscopy images of AF488-labeled scFvLPETG coupled to (PVPON Alk $_{5}$ PEG $_{\text {Alk }}$ capsules. a) Unfunctionalized capsules, b) capsules lacking GGG-PEG-Az incubated with scFvLPETG and sortase, c) capsules with GGG-PEG-Az incubated with SCFV-LPETG but without sortase, d) capsules with GGG-PEG-Az incubated with scFv-LPETG and sortase. Scale bar $=5 \mu \mathrm{m}$.

To evaluate the targeting ability of the enzymatically functionalized polymer carriers, the binding of scFv-functionalized capsules to activated GPIIb/IIIa on thrombi was examined. The specificity of the GPIIb/IIIa-specific scFv $(\operatorname{scFv}(+))$ was compared to a mutated scFv $(\operatorname{scFv}(-))$, which was also cloned with an LPETG tag. GGG-PEG-Az-modified PVPON Alk $_{\text {PEG }} / \mathrm{Plk}_{\text {Alk }}$ capsules were functionalized in the presence of Srt A with the two scFvs to form $\mathrm{scFv}(+)$ and $\mathrm{scFv}(-)$-coated capsules. Unfunctionalized $\mathrm{PVPON}_{\mathrm{Alk}} / \mathrm{PEG}_{\mathrm{Alk}}$ capsules were used as a control to investigate the background binding of the polymer capsules. Thrombi were formed from human platelet rich plasma and incubated with the three types of functionalized and unfunctionalized PVPON/PEG capsules (30 min, $37^{\circ} \mathrm{C}$ ). To analyze the binding, near infrared imaging was used for visualization of the capsule-bound thrombi and the capsules were hence labeled with DyLight 800 (DL800 $\mathrm{Az}$ ). After incubation, the thrombi were washed in phosphate buffered saline (PBS) to remove unattached capsules and the number of bound capsules was expressed qualitatively as fluorescence intensity (Figure 4). The scFv(+)-coated capsules demonstrated highly specific binding to the thrombi with a high fluorescence intensity of 62.5 (the relative fluorescence intensity of untreated thrombi was set at 1). This represents a 9- and 5-fold increase in binding compared to the $\operatorname{scFv}(-)$-coated and uncoated capsules, respectively, indicating the targeting ability of the $\mathrm{scFv}(+)$-coated capsules. Moreover, low levels of nonspecific binding of the control capsules were exhibited, illustrating the low-fouling properties of the PEGylated capsules. This finding is in accordance with other studies with PEGylated capsules. ${ }^{[17]}$ The selective binding of the $\operatorname{scFv}(+)$-coated capsules was also examined by a competitive binding assay. The thrombi were first incubated with excess free $\mathrm{scFv}(+)$ to block the GPIIb/IIIa receptors before exposure to the $\mathrm{scFv}(+)$-coated capsules. Targeting of the $\operatorname{scFv}(+)$-coated capsules to blocked thrombi showed a 13-fold decrease in binding, confirming that the targeting of the $\mathrm{scFv}(+)-$ coated capsules to thrombi is highly specific and is GPIIb/IIIamediated.

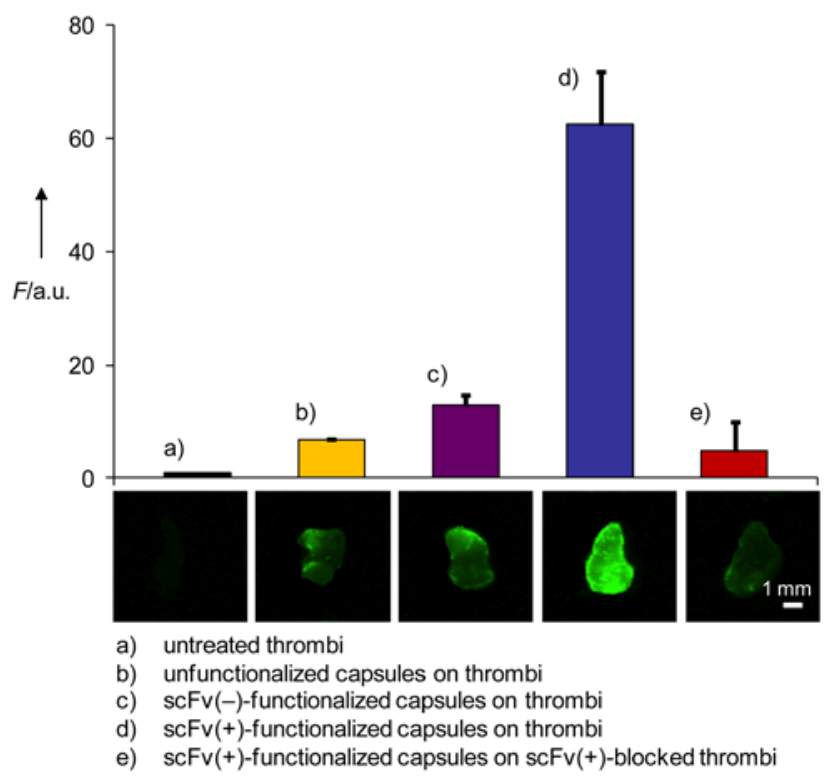

Figure 4. Targeting of scFv-functionalized capsules to thrombi. Relative fluorescence intensities $(F)$ and images of (from left to right) a) untreated and DL800-labeled b) unfunctionalized, c) ScFv(-)functionalized and d) $\mathrm{scFv}(+)$-functionalized $\left(\mathrm{PVPON}_{\mathrm{Alk}}\right)_{5} / \mathrm{PEG}_{\mathrm{Alk}}$ capsule-bound thrombi, and e) a scFv(+)-blocked thrombus attached with $\operatorname{scFv}(+)$-coated capsules. Targeting was conducted for $30 \mathrm{~min}$ at $37^{\circ} \mathrm{C}$ in $\mathrm{pH} 7.2 \mathrm{PBS}$ (with $\mathrm{Ca} / \mathrm{Mg}$ ) and analyzed by near infrared imaging. The fluorescence intensity of untreated thrombi was set at 1.

In summary, we have demonstrated an efficient method for the covalent and site-specific protein functionalization of polymer capsules using Srt A. Due to the precision of the ligation of genetically encoded substrates, this "bio-click" approach allows targeting ligands to be oriented in the right direction with the antigen-binding sites available for binding, thus retaining their bioactivity. The GPIIb/IIIa-specific scFv-functionalized capsules showed highly specific targeting to thrombi, making them promising for anti-thrombotic and thrombolytic therapy. Hence, we expect this 
Srt A-mediated biofunctionalization technique to find a range of applications in functionalizing materials for use in drug delivery as well as diagnostics and imaging.

Received: ((will be filled in by the editorial staff))

Published online on ((will be filled in by the editorial staff))

Keywords: sortase $\cdot$ functionalization $\cdot \mathrm{scFv} \cdot$ targeting $\cdot$ polymer capsules

[1] a) A. P. R. Johnston, G. K. Such, S. L. Ng, F. Caruso, Curr. Opin. Colloid Interface Sci. 2011, 16, 171; b) J. Shi, A. R. Votruba, O. C. Farokhzad, R. Langer, Nano Lett. 2010, 10, 3223.

[2] a) M. M. J. Kamphuis, A. P. R. Johnston, G. K. Such, H. H. Dam, R. A. Evans, A. M. Scott, E. C. Nice, J. K. Heath, F. Caruso, J. Am. Chem. Soc. 2010, 132, 15881; b) D. R. Elias, Z. Cheng, A. Tsourkas, Small 2010, 6, 2460; c) G. von Maltzahn, Y. Ren, J. H. Park, D. Min, V. R. Kotamraju, J. Jayakumar, V. Fogal, M. J. Sailor, E. Ruoslahti, S. N. Bhatia, Bioconjugate Chem. 2008, 19, 1570.

[3] a) M. Kindermann, I. Sielaff, K. Johnsson, Bioorg. Med. Chem. 2004, 14, 2725; b) M. Colombo, S. Mazzucchelli, J. M. Montenegro, E. Galbiati, F. Corsi, W. J. Parak, D. Prosperi, Small 2012, 8, 1492.

[4] a) Y. Kwon, Z. Han, E. Karatan, M. Mrksich, B. K. Kay, Anal. Chem. 2004, 76, 5713; b) I. Sielaff, A. Arnold, G. Godin, S. Tugulu, H. A. Klok, K. Johnsson, ChemBioChem 2006, 7, 194.

[5] S. K. Mazmanian, G. Liu, H. Ton-That, O. Schneewind, Science 1999, 285, 760 .

[6] a) R. G. Kruger, B. Otvos, B. A. Frankel, M. Bentley, P. Dostal, D. G. McCafferty, Biochemistry 2004, 43, 1541; b) H. Ton-That, S. K. Mazmanian, K. F. Faull, O. Schneewind, J. Biol. Chem. 2000, 275,
9876; c) X. Huang, A. Aulabaugh, W. Ding, B. Kapoor, L. Alksne, K. Tabei, G. Ellestad, Biochemistry 2003, 42, 11307.

[7] S. Samantaray, U. Marathe, S. Dasgupta, V. K. Nandicoori, R. P. Roy, J. Am. Chem. Soc. 2008, 130, 2132.

[8] X. Guo, Q. Wang, B. M. Swarts, Z. Guo, J. Am. Chem. Soc. 2009, 131, 9878.

[9] S. Pritz, Y. Wolf, O. Kraetke, J. Klose, M. Bienert, M. Beyermann, J. Org. Chem. 2007, 72, 3909

[10] a) M. W. Popp, J. M. Antos, G. M. Grotenbreg, E. Spooner, H. L. Ploegh, Nat. Chem. Biol. 2007, 3, 707; b) T. Tanaka, T. Yamamoto, S. Tsukiji, T. Nagamune, ChemBioChem 2008, 9, 802; c) H. T. Ta, S. Prabhu, E. Leitner, F. Jia, D. von Elverfeldt, K. E. Jackson, T. Heidt, A. K. N. Nair, H. Pearce, C. von Zur Muhlen, X. Wang, K. Peter, C. E. Hagemeyer, Circ. Res. 2011, 109, 365.

[11] a) R. Parthasarathy, S. Subramanian, E. Boder, Bioconjugate Chem. 2007, 18, 469; b) L. Chan, H. F. Cross, J. K. She, G. Cavalli, H. F. P. Martins, C. Neylon, PLoS one 2007, 2, 1164.

[12] a) P. Holliger, P. J. Hudson, Nat. Biotechnol. 2005, 23, 1126; b) C. E. Hagemeyer, M. Schwarz, K. Peter, Semin. Thromb. Hemost. 2007, 33, 185.

[13] M. Schwarz, G. Meade, P. Stoll, J. Ylanne, N. Bassler, Y. C. Chen, C. E. Hagemeyer, I. Ahrens, N. Moran, D. Kenny, D. Fitzgerald, C. Bode, K. Peter, Circ. Res. 2006, 99, 25.

[14] C. R. Kinnane, G. K. Such, G. Antequera-Garcia, Y. Yan, S. J. Dodds, L. M. Liz-Marzan, F. Caruso, Biomacromolecules 2009, 10, 2839.

[15] M. K. M. Leung, G. K. Such, A. P. R. Johnston, D. P. Biswas, Z. Zhu, Y. Yan, J. F. Lutz, F. Caruso, Small 2011, 7, 1075.

[16] G. Sauerbrey, Z. Physik. 1959, 155, 206.

[17] U. Wattendorf, O. Kreft, M. Textor, G. B. Sukhorukov, H. P. Merkle, Biomacromolecules 2008, 9, 100 . 
Entry for the Table of Contents (Please choose one layout)

Layout 2:

Enzymatic Biofunctionalization

Melissa K. M. Leung, Christoph E. Hagemeyer, Angus P. R. Johnston, Catalina Gonzales, Marloes M. J. Kamphuis, Katie Ardipradja, Georgina K. Such, Karlheinz Peter, and Frank Caruso* Page - Page

Bio-Click Chemistry: Enzymatic Functionalization of PEGylated Capsules for Targeting Applications
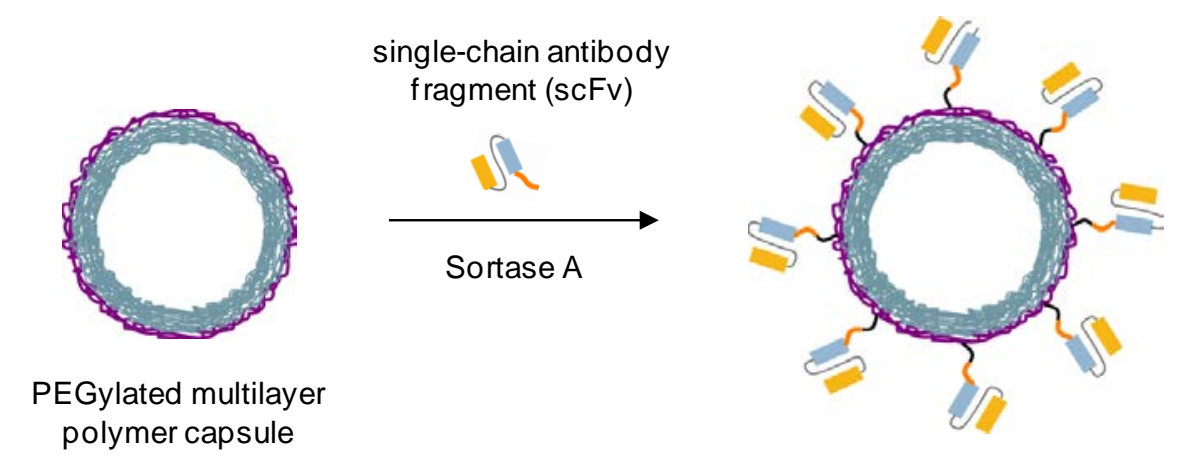

scFv-functionalized PEGylated capsule

The enzyme Sortase A is used as a catalyst for the functionalization of PEGylated capsules with an activated platelet-specific single-chain antibody (scFv). This enzymatic method allows fast, covalent and site-directed functionalization of delivery vehicles in mild conditions. Activated platelet-specific scFv-coated PEGylated capsules exhibit highly selective binding to thrombi (platelet aggregates), suggesting their potential for thrombosis therapy. 


\section{University Library}

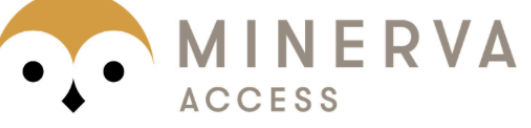

A gateway to Melbourne's research publications

Minerva Access is the Institutional Repository of The University of Melbourne

\section{Author/s:}

Leung, MKM;Hagemeyer, CE;Johnston, APR;Gonzales, C;Kamphuis, MMJ;Ardipradja,

K;Such, GK;Peter, K;Caruso, F

Title:

Bio-Click Chemistry: Enzymatic Functionalization of PEGylated Capsules for Targeting Applications

Date:

2012-01-01

Citation:

Leung, M. K. M., Hagemeyer, C. E., Johnston, A. P. R., Gonzales, C., Kamphuis, M. M. J., Ardipradja, K., Such, G. K., Peter, K. \& Caruso, F. (2012). Bio-Click Chemistry: Enzymatic Functionalization of PEGylated Capsules for Targeting Applications. ANGEWANDTE CHEMIE-INTERNATIONAL EDITION, 51 (29), pp.7132-7136. https://doi.org/10.1002/ anie.201203612.

Persistent Link:

http://hdl.handle.net/11343/123310 